\title{
Brownian motion in strongly fluctuating liquid
}

\section{Mouvement brownien en liquide fortement fluctuant}

\author{
Daniel Beysens ${ }^{1}$ \\ ${ }^{1}$ Directeur de Recherches Honoraire - Physique et Mécanique des Milieux Hétérogènes- École Supérieure de Physique \\ et Chimie Industrielles de la Ville de Paris
}

\begin{abstract}
In this paper is reported how the Brownian motion of colloids (beads) immersed in a liquid can be affected by the local properties of the fluid and how, in turn, it can alter them. Near- critical binary mixtures are known to exhibit important concentration fluctuations when nearing their liquid-liquid critical point (CP). Photon-beating spectroscopy measurements show that colloids immersed in such a critical mixture of isobutyric acid and water exhibit a slow-down of their Brownian motion due to the fluctuation-induced critical enhancement of viscosity. However, as the mixture temperature is brought close to the CP temperature, the fluctuations lifetime becomes large enough such that the fluctuations can be in turn strongly deformed by the shear flow around the colloids. Mean-field behavior, where the influence of fluctuations can be ignored, follows. Viscosity does not increase anymore and colloids motion ceases to slow down.

KEYWORDS. Brownian motion, liquid mixture, critical point, colloïds, fluctuations.
\end{abstract}

\section{Introduction}

Brownian motion of small particles (colloids) immersed in a liquid has been studied for more than a century since the works of Einstein and Perrin ${ }^{\mathrm{i}}$, ii. Recently, the use of modern techniques (fast videos, optical tweezers) with high spatial and temporal resolution has given rise to the precise determination of instantaneous velocityii-v. For the latter, the hydrodynamic coupling between the colloid and the fluid has been seen to dominate the dynamics of velocity fluctuations. In particular, the equipartition theorem is verified only if one considers the "added mass" of fluid accounting for the inertia of the displaced fluid in the colloid motion ${ }^{\mathrm{vi}}$.

Local hydrodynamics around the colloids can thus affect their Brownian motion. Hydrodynamic coupling is enhanced when nearing a critical point where the local fluctuations of the order parameter (concentration fluctuations in a liquid mixture near its miscibility critical point) affect the fluid resistance to flow, resulting in viscosity enhancement ${ }^{\mathrm{vii}}$. When a colloid is immersed in a binary liquid near its liquid-liquid critical point, its Brownian motion can therefore be seriously affected by the fluctuation-induced enhancement of viscosity. However, the shear flow around the colloid can in turn reduce the local concentration fluctuations, resulting in saturation of the friction (see below section 2.2). This complex hydrodynamic process disturbs the Brownian motion of colloids as shown by the result of photon beating light scattering experiments performed in the mixture of isobutyric acid and water above its critical point - where it is homogeneous.

This paper is organized as follows. Firstly, the bases of critical point phenomena, shear flow effects and photon beating technique are exposed. Secondly the experimental methods are described and then the results are exposed and discussed.

\footnotetext{
${ }^{1}$ Physique et Mécanique des Milieux Hétérogènes, CNRS, ESPCI, PSL Research University, Sorbonne Université, Sorbonne Paris Cité, Paris, France - e-mail : daniel.beysens@espci.fr 


\section{Theoretical background}

\subsection{Miscibility critical point in binary mixtures}

The vicinity of second-order phase transition (critical point) exhibit a number of universal, scaled behavior $^{\text {viii, ix }}$. Fluids near their liquid-vapor critical point and partially miscible binary mixtures near their liquid-liquid critical point (the upper point in the temperature-density or concentration phase diagram) exhibit the same features as they belong to the same universality class. The class representative is the magnetic Ising model with magnetization $M$ as the order parameter. In binary fluids, $M$ is the concentration difference $c-c_{c}$, with $c$ the concentration of one of the components and $c_{c}$ the critical concentration. This class is characterized by the dimensionalities of space $D=3$ and dimensionality of order parameter, $d=1$. An important aspect of the critical region is that most of the anomalies of the thermodynamic and transport properties can be set in the form of scaled, universal power laws with respect to the critical point coordinates. For instance, the correlation length of concentration fluctuations, $\xi$, asymptotically diverges with the reduced temperature $\varepsilon=\frac{T-T_{C}}{T_{C}}$ (here $T$ is absolute temperature and $T_{c}$ the critical temperature) along the $c=c_{c}$ thermodynamic path, as

$$
\xi=\xi_{0} \epsilon^{-v}
$$

Here $v$ is a universal exponent and $\xi_{0}$ is a system-dependent critical amplitude. The value of critical exponents depends on $D$. Above an upper space critical dimensionality $D_{c}=4$, the effect of fluctuations on divergence can be neglected, mean field approach is relevant and $v=0.5$. For the class of fluids where the space dimensionality $D=3<D_{c}$, fluctuations have to be accounted for, which renormalizes the exponent value to $v=0.630^{\text {viii, ix. }}$

The lifetime of concentration fluctuation corresponds to the diffusion of a component on lengthscale $\xi$. The fluctuation indeed vanishes by a diffusion process, with a diffusion coefficient $D_{\xi}$ which can be related to the Brownian diffusion of a cluster of size $\xi^{\text {vii }}$. With $k_{B}$ the Boltzmann constant and $\eta$ the liquid mixture shear viscosity, the diffusion constant then reads as

$$
D_{\xi}=\frac{k_{B} T}{6 \pi \eta \xi}
$$

Diffusion on length $\xi$ occurs on time $\tau$ such as

$$
\tau=D_{\xi}{ }^{-1} \xi^{2}=\left(\frac{6 \pi \eta}{k_{B} T}\right) \xi^{3}=\left(\frac{6 \pi \eta \xi_{0}^{3}}{k_{B} T}\right) \epsilon^{-3 \nu}
$$

Shear viscosity, defined as the ratio of the shear stress to an infinitesimally small velocity gradient or shear flow $S$, the so-called Newton's law for fluids, is also affected by the coupling with critical fluctuation $^{\text {vii }}$. Viscosity exhibits a (weak) divergence such as

$$
\eta=\eta_{B}(T) \epsilon^{-Y}
$$

Here the critical exponent is $Y=0.04$ and $\eta_{B}(T)$ is a thermally activated regular (background) contribution of Arrhenius form. With $E$ the activation energy and $\eta_{0}$ the amplitude:

$$
\eta_{B}(T)=\eta_{0} \exp \left(\frac{E}{k_{B} T}\right)
$$

\subsection{Effect of shear flow}

Spatial fluctuations near the critical point can be strongly deformed by external influences like hydrodynamics. Shear flow alters the spatial fluctuations ${ }^{\text {vii, } x}$ when the shear-induced fluctuation deformation, $l$, during the fluctuation lifetime $\tau$, becomes larger than the fluctuation correlation length $\xi$, that is $l=S \xi \tau>\xi$, or 
It means that the fluctuation lifetime is large enough to "feel" the shear. The above condition Eq. 6, when considering the fluctuation lifetime expression Eq. 3, corresponds to having a cross-over correlation length, $\xi_{c}$, above which fluctuations become strongly deformed by shear:

$$
\xi_{c}=\left(\frac{k_{B} T}{6 \pi \eta}\right)^{1 / 3} S^{1 / 3}
$$

When nearing a critical point on the thermodynamic path $c=c_{c}$ by changing temperature, the wellknown "critical slowing down" where $\tau$ increases, ensures that the condition Eq. 6 will be eventually fulfilled. From Eq. 3 derives a condition on temperature, separating two regions of low and strong shear on both sides of $\varepsilon=\varepsilon_{c}$ where

$$
\varepsilon_{c}=\left(\frac{6 \pi \eta \xi_{0}^{3} S}{k_{B} T}\right)^{1 / 3 v}
$$

The reduction of fluctuations in the strong shear region is such that mean-field behavior, where fluctuations can be ignored, becomes relevant very near the $\mathrm{CP}^{\mathrm{vii}, \mathrm{x}}$. In other words, the upper critical dimensionality is lowered (to $D_{c}=2.4^{\mathrm{vii}}$ ), below the space dimensionality $D=3$. As a matter of fact, viscosity in the mean-field approximation does not show any more a divergence and levels off $\mathrm{f}^{\mathrm{vi} i \mathrm{xi}}$.

\subsection{Colloid Brownian motion and shear flow}

The mean value of the colloid velocity can be inferred from the equipartion theorem to be, for each Cartesian component of velocity:

$$
\langle V\rangle=\sqrt{\frac{k_{B} T}{m^{*}}}
$$

In a liquid, the inertial mass of an object has to be modified by the inertia of the displaced fluid ${ }^{\mathrm{vi}}$, (the so-called added mass $m_{a}$ ) which is half of the mass of the displaced fluid ${ }^{\mathrm{vi}}$. For a sphere of radius $a$ immersed in a fluid with density $\rho_{f}, m_{a}=\frac{2 \pi}{3} a^{3} \rho_{f}$. The sphere mass being $m=\frac{4 \pi}{3} a^{3} \rho_{s}, \quad$ with sphere density $\rho_{s}$, the effective mass $m^{*}$ reads as

$$
m^{*}=\frac{2 \pi}{3} a^{3}\left(\rho_{f}+2 \rho_{s}\right)
$$

When dealing with spherical colloids in Brownian motion, the Reynolds number is generally very low (see below Eq. 18 and its evaluation), indicating that the flow is dominated by viscous effects around the sphere. Stokes damping can thus be successfully assumed. Hydrodynamics can be considered as stationary as it corresponds to a diffusive behavior over times longer than the viscous time $a^{2} \rho_{f} / \eta \approx 2.6 \mathrm{~ns}$ for the colloids used here (see Table 1 for the numerical values). This time is much shorter than the binary liquid fluctuation time $\tau>50$ ns (values from Table 1) in the studied temperature range.

The stationary velocity field around a sphere with velocity $V$ at small Reynolds number is axisymmetric and is well-known ${ }^{\mathrm{xii}}$. In polar coordinates $(r, \theta)$, the components of fluid velocity, $v_{r}$ along $r$, and $v_{\theta}$, perpendicular to $r$, are

$$
v_{r}(r, \theta)=-V \cos \theta\left[-\frac{3}{2} \frac{a}{r}+\frac{1}{2}\left(\frac{a}{r}\right)^{3}\right]
$$

and

$$
v_{\theta}(r, \theta)=-V \sin \theta\left[\frac{3}{4} \frac{a}{r}+\frac{1}{4}\left(\frac{a}{r}\right)^{3}\right]
$$


The shear rate along $r$ can be deduced by derivating Eq. 11 with respect to $r$ for $r / a>1$. The maximum shear flows $S$ corresponds to $v_{r}$ at $\theta=0$ at $r / a=\sqrt{2}, S_{r}=\left(\frac{d v_{r}}{d r}\right)_{\max }=\frac{3}{8}$. The macimum shear rate perpendicular to $r$ correponds to $v_{\theta}$ at $\theta=\pi / 2$ and $r=a, S_{\theta}=\left(\frac{d v_{\theta}}{d r}\right)_{\max }=\frac{3}{2} \frac{V}{a}$. One will thus consider in the following the mean value

$$
S \approx \frac{V}{a}
$$

With the numerical values of Table 1 , one finds $V=<V>=3.5 \times 10^{-2} \mathrm{~ms}^{-1}$, giving $S=4.4 \times 10^{5} \mathrm{~s}^{-1}$.

\subsection{Light scattering (photon beating spectroscopy)}

Colloids immersed in a liquid lead to concentration fluctuations and thus to refractive index fluctuations, which scatter light. The dynamics of these fluctuations is driven by the diffusion of colloids and can be analyzed by recording the frequency spectrum of the scattered light (Figure 1) or equivalently its autocorrelation function. In the photon beating homodyne arrangement used here the scattered light interferes with itself and the autocorrelation function is a decaying exponential function with typical time $\tau_{0}$ such as ${ }^{\text {xii }}$

$$
\tau_{0}=\frac{1}{2 D q^{2}}
$$

Here $D$ is the colloid diffusion coefficient

$$
D=\frac{k_{B} T}{6 \pi \eta a}
$$

and

$$
q=2 K_{0} \sin \frac{\alpha}{2}
$$

is the transfer wavevector corresponding to the scattering angle $\alpha$ (see Figure 1). The incident light wavevector $K_{0}$ reads, with $n$ the liquid refractive index and $\lambda_{0}$ the wavelength of incident light:

$$
K_{0}=\frac{2 \pi n}{\lambda_{0}}
$$

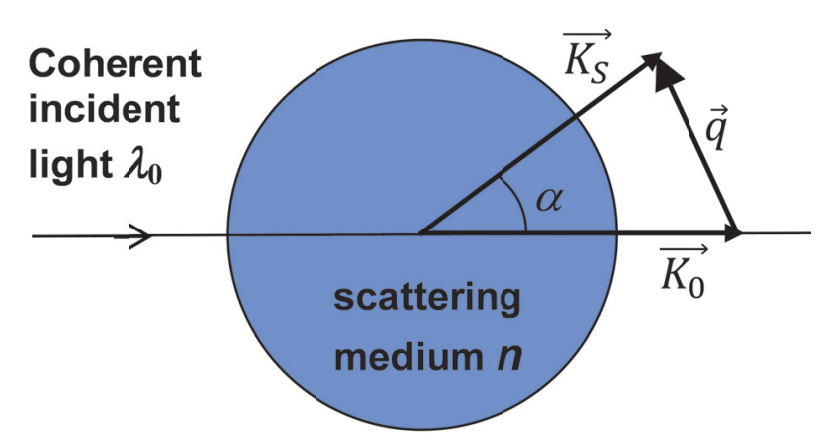

Figure 1. Light scattering arrangement. $\overrightarrow{K_{0}}:$ Incident light wavevector; $\overrightarrow{K_{S}}$ : Scattered light wavevector; $\vec{q}=\overrightarrow{K_{S}}-$ $\overrightarrow{K_{0}}$ : Transfer wavevector; $n$ : Refractive index; $\lambda_{0}$ : Incident light wavelength.

\section{Experiments and methods}

The colloids considered here are latex beads from Dow Chemicals whose characteristics are listed in Table 1. The latex bead suspensions are composed mainly of polymer particles and water, with small amounts of surfactant, sodium bicarbonate and potassium sulfate. A typical suspension contains the following: water $>69.0 \%$, polymer $30.0 \%$, surfactant $0.1-0.5 \%$, inorganic salts $0.2 \%$. A very dilute 
solution with water was added to the mixture of isobutyric acid and water. Photon beating spectroscopy of beads far from $T_{c}$ gives $a=8.0 \times 10^{-8} \mathrm{~m}$ from Eqs. 14, 15. It is implicitly assumed that the flow is dominated by viscosity around the colloids, as assessed by the Reynolds number $<<1$, see Eq.18.

The binary mixture of isobutyric acid and water exhibits an upper critical point with a critical mass fraction of isobutyric acid $c_{c}=0.3889$ and temperature $T_{c}=301 \mathrm{~K}$ [14]. A precise value is not necessary, as minutes quantities of impurities can shift $T_{c}$. What really matters is the temperature difference $T-T_{c}$, with $T_{c}$ experimentally determined for each sample. The refractive index at the critical point is $n_{c}=$ 1.36105 for the laser wavelength $\lambda_{0}=632.8 \mathrm{~nm}$. With a mean temperature dependence $(d n / d T) \approx-$ $2.5 \times 10^{-4} \mathrm{~K}^{-1}$ and a studied temperature range $T-T_{c} \approx 20 \mathrm{~K}$, the refractive index thermal variation $\Delta n<$ $5 \times 10^{-3}$ and can be neglected. In the following the value $n=1.361$ will be used (Table 1). Concerning density, its temperature variation will be also neglected and the value at $T_{c}, \rho_{f}=0.99^{\mathrm{xiv}, \mathrm{xv}}$ is reported in Table 1. Isobutyric acid of quality better than 99.99 mol\% was used without further purification. Ultrapure water from Millipore purification treatment device was used. The experimental isobutyric acid mass fraction used was $0.3887 \pm 0.0002$, close enough to $c_{c}$.

The mixture with colloids is set in a sealed glass sample, itself put in a thermostat with temperature regulation to within $\pm 10^{-3} \mathrm{~K}$. The beam of a $10 \mathrm{~mW}$ He-Ne polarized laser is slightly focused in the sample. Collimated light scattered at $\alpha=90^{\circ}$ is recorded by a photomultiplier, whose homodyne amplified signal is then analyzed by a correlator. The critical temperature is determined within $\pm 5 \mathrm{mK}$ by decreasing temperature step by step and looking at the phase separation process on a screen in front of the sample, perpendicular to the laser beam. The value $T_{c}=(301.130 \pm 0.005) \mathrm{K}$ was found. Note that the signal due to light scattered by colloids is more important than the signal due to the mixture critical fluctuations, preventing from interfering in the analysis.

\begin{tabular}{c|c|c|c|c|c|c|c|c} 
& $\begin{array}{c}\text { Correlation } \\
\text { length amplitude }\end{array}$ & $\begin{array}{c}\text { Refrac- } \\
\text { tive index }\end{array}$ & $\begin{array}{c}\text { Incident } \\
\text { wave- } \\
\text { length }\end{array}$ & $\begin{array}{c}\text { Fluid } \\
\text { density }\end{array}$ & $\begin{array}{c}\text { Bead } \\
\text { density }\end{array}$ & $\begin{array}{c}\text { Bead } \\
\text { radius }\end{array}$ & \multicolumn{2}{|c}{$\begin{array}{c}\text { Shear viscosity } \\
\eta_{0} \exp \left(\frac{E}{k_{B} T}\right) \epsilon^{-0.04}\end{array}$} \\
\cline { 2 - 8 } & $\xi_{0}(\mathrm{~nm})$ & $n$ & $\lambda_{0}(\mathrm{~nm})$ & $\rho_{f}\left(\mathrm{~kg} \cdot \mathrm{m}^{-3)}\right.$ & $\rho_{s}\left(\mathrm{~kg} \cdot \mathrm{m}^{-3)}\right.$ & $a(\mathrm{~nm})$ & $\eta_{0}\left(\times 10^{-6}\right.$ Pa.s $)$ & $E\left(\times 10^{-20} \mathrm{~J}\right)$ \\
\hline $\begin{array}{c}\text { IW liquid } \\
\text { mixture }\end{array}$ & $0.3625^{(\text {a) }}$ & $1.361^{(\mathrm{b})}$ & 632.8 & $990^{(\mathrm{b})}$ & - & - & $1.4422^{(\mathrm{b})}$ & $3.007^{(\mathrm{b})}$ \\
\hline $\begin{array}{c}\text { Latex } \\
\text { bead }\end{array}$ & - & - & - & - & $1053^{(\mathrm{c})}$ & $80^{(\mathrm{b})}$ & - & -
\end{tabular}

Table 1. Useful data. (a) Ref. (xx). (b) See text. (c) Ref. (xxi).

\section{Results and discussion}

The procedure to collect data is to vary temperature in logarithmic steps of the difference $T-T_{c}$ in order to generate a uniform density of measurements when looking at the data in log-log scale. The correlation time data are reported in Supplementary Material. Log-log scale indeed evidences power laws (see Figure 2). 43 correlation times data have been obtained in the temperature range [0.01920.6] K. The use of Eq. 9 with laminar flow around the colloid and Stokes damping can be tested. Equation 9 gives a velocity $V=3.6 \times 10^{-2} \mathrm{~m} . \mathrm{s}^{-1}$, using the data of Table 1 . With liquid mixture viscosity $\sim 2.5 \times 10^{-3}$ Pa.s. (Table 1 ), the associated Reynolds number is indeed very low:

$$
\operatorname{Re}=\frac{\rho_{f} V a}{\eta} \sim 1 \times 10^{-3}
$$

From the correlation time $\tau_{0}$ an experimental diffusion constant can be obtained by using Eq. 14 . The corresponding values are drawn in Figure 2. In the same Figure the data are compared with the 
calculated Brownian motion diffusion constant using viscosity data, Eq. 15. Viscosity is deduced from the capillary flow measurements of Ref. (xvi), whose mean shear rate as estimated in Ref. (xi) leads to ignore the data at $T-T_{c}<3 \times 10^{-2} \mathrm{~K}$ as corresponding to the strong shear region (see above Section 2.2). A fit of the viscosity data is made to Eq. 4, which allows the background contribution to $D$ to be evidenced (Eq. 5). One finds $\eta_{0}=(1.422 \pm 0.25) \times 10^{-6}$ Pa.s. and $E=(3.007 \pm 0.07) \times 10^{-20} \mathrm{~J}$, with a linear correlation coefficient $R=0.999$ and $\chi^{2}=3.89 \times 10^{-9}$.

Two regions of high and low shear are clearly evidenced in Figure 2 depending on the value of $S \tau$ with respect to 1 , or equivalently $T-T_{c}$ with respect to $3.5 \mathrm{~K}$ (see Eq. 3 and numerical values in section 2.3, $S=4.4 \times 10^{-5} \mathrm{~s}^{-1}$ ). The calculated $D$ values using the background viscosity clearly do not fit the data . Using the total viscosity Eq. 4 , in the low shear region $\left(T-T_{c}>3.5 \mathrm{~K}\right)$, both diffusion constants take the same values accounting for the fluctuation- induced enhancement of viscosity with respect to temperature. It means that the Brownian motion of the colloids follows the viscosity enhancement, corresponding to an augmentation of the friction forces. The contribution of fluctuations is made apparent in this low shear region when one compares the values of the experimental diffusion coefficient with the value inferred from the background viscosity. In the high shear region $\left(T-T_{c}<3.5\right.$ $\mathrm{K})$, the experimental diffusion constant saturates, in relation with the fluctuations deformation leading to a saturation of the viscosity enhancement. In other words, mean-field where the influence of fluctuations is disregarded becomes relevant, as noted in Section 2.2.

It is interesting to compare those data with other data obtained with PTFE (Teflon) microspheres of $1.15 \mu \mathrm{m}$ radius immersed in the same isobutyric critical mixture at critical concentration ${ }^{\text {xvii }}$. The data have been analyzed to determine viscosity assuming Eqs. 14, 15. Eight data have been obtained in the range $T-T_{c}=[0.6-6.8] \mathrm{K}$. The transition high-low shear is expected to occur at $T-T_{c}=0.06 \mathrm{~K}$, well below the investigated temperature range.

It is worthy to note that, in contrast to other colloids immersed in different liquid mixtures (e.g. silica beads in lutidine-water mixtures ${ }^{\text {xviii, }}$ xix , no colloid aggregation phenomena nor preferential component adsorption were observed onto the colloids. The latter would have increase $a$, that is, decrease $D$, an effect in opposition to the saturation effect observed in Figure 2.

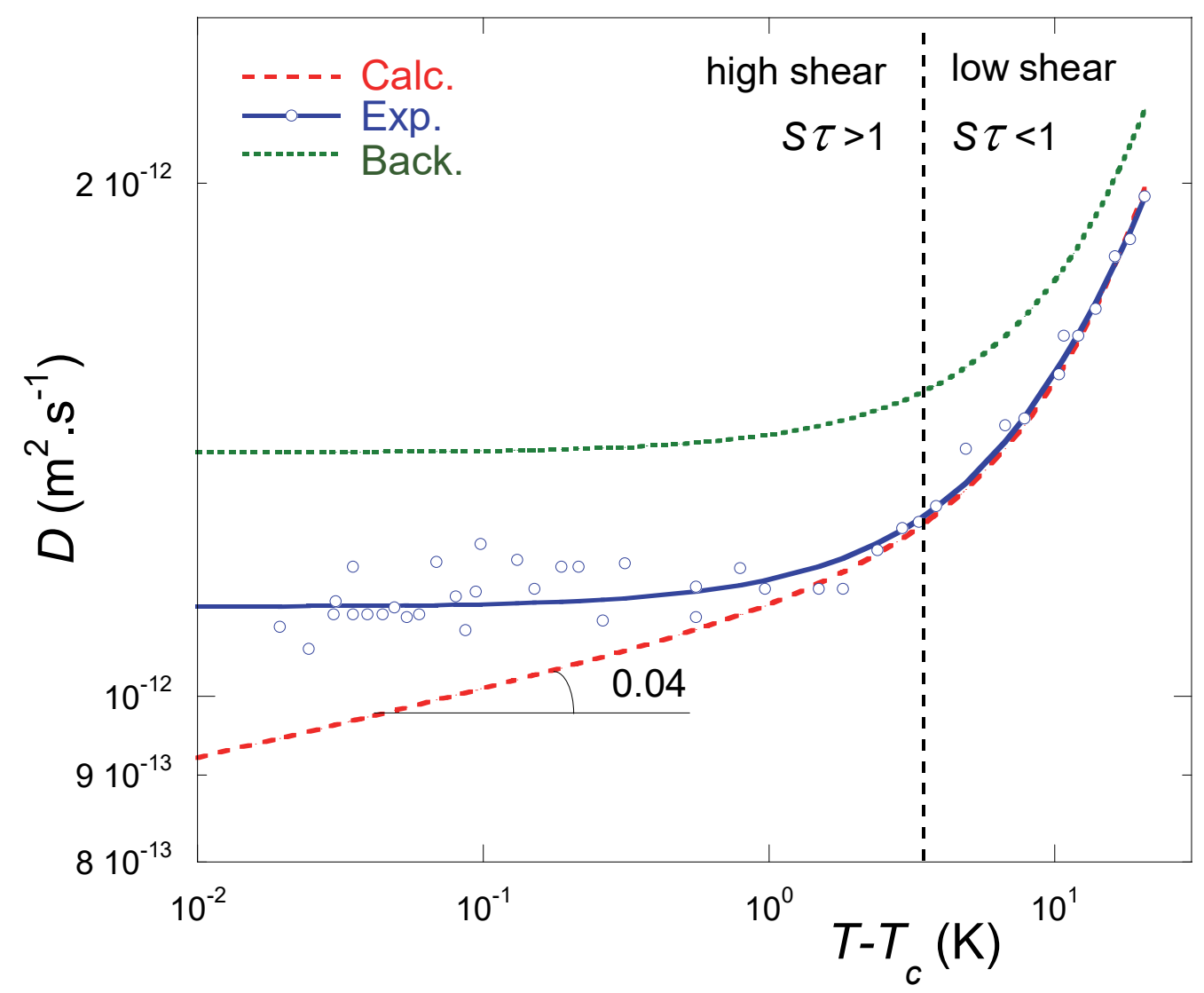


Figure 2. Temperature dependence of the diffusion constant $D$ (log-log plot) as obtained from the experimental correlation times (open circles, exp.), Brownian motion diffusion constant using full viscosity (Eq. 4) from Ref. (xvi) (interrupted line, calc.) or using only background viscosity from a fit to Eq. 5 (see text; dotted line, back.). The full line is a smoothing function of the data.

\section{Concluding remarks}

Photon beating light scattering experiments as described in this paper show that the Brownian motion of colloids (beads) immersed in a liquid is closely related to the hydrodynamics around the colloids. This behavior is highlighted in a near-critical liquid mixture which undergoes large concentration fluctuations. In such a liquid, diffusion of immersed colloids is indeed seen to be sensitive to the augmentation of viscosity related to the critical enhancement of concentration fluctuations. However, as the liquid mixture is brought close enough to the critical point where fluctuations increase, it turns out that the concentration fluctuations are altered by the shear flow around the colloids. It follows a deformation of fluctuations and a saturation of the viscosity enhancement, resulting in a mean-field behavior which ignores the effect of fluctuations. Such results show that the Brownian motion of particles in a dense fluid can either reflect the fluid properties or modify them or both.

\section{Acknowledgments}

I gratefully thank S. Perleaux for help in the measurements and A. Mongruel for a critical reading of the manuscript.

\section{References}

i Einstein A.: On the Motion of Small Particles Suspended in Liquids at Rest Required by the Molecular-Kinetic Theory of Heat, Ann. Phys. (Berlin) 17 549-560(1905). (In German).

ii Perrin J. : Mouvement brownien et réalité moléculaire, Ann. Chim. Phys. 18, 5-104 (1909). (In French).

iii Li T., Kheifets S., Medellin D., Raizen M. G.: Measurement of the Instantaneous Velocity of a Brownian Particle, Science 328, 1673-1675 (2010).

iv Huang R., Chavez I., Taute K.M., Lukić B., Jeney S., Raizen M.G., Florin E.-L.: Direct observation of the full transition from ballistic to diffusive Brownian motion in a liquid, Nature Physics 7, 576-579 (2011).

v Kheifets S., Simha A, Melin K., Li T., Raizen M.G.: Observation of Brownian Motion in Liquids at Short Times: Instantaneous Velocity and Memory Loss Science 343,1493-1496 (2014).

vi Zwanzig R., Bixon M.: Compressibility effects in the hydrodynamic theory of Brownian motion, J. Fluid Mech. 69, 21-25 (1975).

vii Onuki A. (2002) Phase transition dynamics. Cambridge University Press and Refs. therein.

viii Stanley H. E. (1987) Introduction to Phase Transitions and Critical Phenomena. Oxford University Press and refs. therein.

ix Zappoli B., Beysens D., Garrabos Y. (2015) Heat Transfers and Related Effects in Supercritical Fluids. Fluid Mechanics and Its Applications. Volume 108 Springer Netherlands and refs. therein.

x Beysens D., Gbadamassi M.: Light-scattering study of a critical mixture with shear flow, J. de Physique Lett. 40, L565-567 (1979).

xi Oxtoby W.: Shear gradient dependence of the viscosity of critical mixtures, Physics Letters A 50, 459-460 (1975).

xii Happal J., Brenner H. (1963) Low Reynolds number Hydrodynamics. Kluwer Academic Publisher, $1^{\text {st }}$ edition.

xiii Cummins H. (Editor) (1974) Photon Correlation and Light Beating Spectroscopy. Springer, Nato Science Series B.

xiv Toumi A., Bouanz M.: Volumetric and refractive index properties of isobutyric acid—water binary mixtures at temperatures ranging from 300.15 to $313.15 \mathrm{~K}$, Journal of Molecular Liquids 139 55-60 (2008).

Xv Jacobs D. T., Greer S.C.: Amplitude of the anomaly in the mass density near a liquid-liquid critical point. Phys. Rev. E 54, 5358-5363 (1996).

xvi Allegra J. C., Stein A., Allen G.F.: Tracer Diffusion and Shear Viscosity for the System Isobutyric Acid-Water near the Critical Mixing Point, J. Chem. Phys. 55, 1716 (1971). 
xvii Carini Jr., G. Maisano G., Migliardo P., Wanderlingh F.: Comparative measurements of viscosity near the critical point, Phys. Rev. A 11, 1755-1762 (1975).

xviii Beysens D., Esteve D., Adsorption Phenomena at the Surface of Silica Spheres in a Binary Liquid Mixture, Phys. Rev. Lett. 54, 2123-2126 (1985).

xix Yabunaka S., Onuki A.: Critical adsorption profiles around a sphere and a cylinder in a fluid at criticality: Local functional theory, Physical Review E 96, 032127 (2017) and refs. therein.

xx Beysens D., Bourgou A., Calmettes P.: Experimental determinations of universal amplitude combinations for binary fluids. I. Statics, Phys. Rev. A 26, 3589-3609 (1982).

xxi Giddings J. C., Ho J.: Accurate Measurement of Density of Colloidal Latex Particles by Sedimentation Field-Flow Fractionation, Langmuir 11, 2399-2404 (1995). 